\title{
Les enjeux économiques des politiques de prévention du risque climatique
}

\author{
Olivier Godard \\ directeur de recherche au CNRS
}

Les risques climatiques planétaires résultant des émissions anthropiques de gaz à effet de serre (GES) offrent à l'actualité diplomatico-scientifique un thème récurrent depuis une vingtaine d'années. Sur le terrain scientifique, des conférences internationales comme celle de Genève en 1979 ou de Toronto en 1988 ont joué un rôle marquant. Une organisation spécifique de l'expertise mondiale a vu le jour la même année 1988, avec la création du Groupe d'experts intergouvernemental sur l'évolution du climat (GIEC) à l'initiative de l'Organisation météorologique mondiale et du Programme des Nations Unies pour l'environnement. Ensemble, ces éléments ont été des catalyseurs pour le développement de la recherche mondiale dans ce domaine autant que des moyens de faire connaître les résultats scientifiques aux responsables politiques et économiques et à l'opinion internationale. Sur le terrain diplomatique, l'événement majeur fut l'adoption à Rio de Janeiro en juin 1992 de la Convention-cadre sur les changements climatiques visant à prévenir une interaction dangereuse des activités humaines avec le climat de la planète. C'est de ce texte fondateur qu'est né un nouveau processus diplomatique continu, jalonné par les conférences internationales comme celle de Berlin (1995), Kyoto (décembre 1997) et Buenos Aires (novembre 1998).

Un équipage à double attelage est désormais lancé et, si sa vitesse de progression peut sembler bien lente à certains observateurs impatients, cet équipage paraît désormais bien difficile à arrêter, comme l'ont expérimenté à leurs dépens les puissants lobbies industriels charbonniers, pétroliers ou automobiles, notamment américains, qui pensaient être en mesure de stopper le convoi, voire de le pousser dans le ravin, au détour de Kyoto.

Le problème de l'effet de serre est-il seulement affaire de mariage entre les sciences de la nature et la politique internationale ? Ce serait aller trop vite en besogne et oublier tous les tiers dont on ne se passe pas sans dommage. Ainsi, la sociologie de l'expertise jette une lumière pénétrante sur les interactions entre science et processus politique et pose des interrogations difficiles à balayer de la main sur les conditions institutionnelles de prise en charge d'une question comme les risques climatiques planétaires (Roqueplo, 1993). L'analyse économique apporte, quant à elle, la construction de repères objectifs dans l'ordre des valeurs et des choix, pointe des enjeux économiques insoupçonnés et aide à saisir la nature des difficultés à surmonter du point de vue de l'organisation de l'action.

Le point de vue économique s'est révélé à ce point essentiel que le GIEC lui a consacré un de ses trois groupes de travail, le groupe III. Le rapport remis par ce dernier fin 1995 (Bruce et al, 1997) présente une somme remarquable sur les raisonnements, méthodes et évaluations que met en œuvre une démarche économique pour affronter un aussi vaste problème que l'effet de serre. Symétriquement, les risques climatiques planétaires lancent de 
multiples défis à l'analyse économique, obligée par la force des choses de se rendre aux confins de son territoire et de mettre en doute certains des outils qu'elle s'était accoutumée à manier de façon routinière, comme par exemple l'actualisation ou l'analyse coûts-avantages. Il y a là une épreuve de pertinence pour les concepts et les méthodes développés notamment en économie publique, en économie de l'environnement, en théorie des jeux et en théorie de la décision. de traits :

C'est que les risques climatiques planétaires combinent de façon inédite un ensemble

- le caractère planétaire, qui impose un niveau de coordination internationale de l'action rarement atteint jusqu'ici entre des pays que distinguent des différences de tous ordres, à la fois géographiques, culturelles, économiques et historiques, sans qu'une autorité supérieure de type étatique ne soit encore en mesure d'exercer une discipline ;

- la dimension du long terme et de la faible réversibilité, à l'échelle des temps humains et économiques, des phénomènes physiques en jeu; les scénarios du GIEC (Bruce et al, 1997) et le débat international (par exemple Wigley et al, 1996 ; Wigley, 1997 ; Ha-Duong et al., 1997) envisagent des horizons tout à fait inhabituels pour cadrer la prise de décision politique et économique (avec les années 2150 ou 2200 pour années terminales des exercices de simulation, 2030 ou 2050 comme horizon intellectuel de référence et 20002010 comme horizon pratique des décisions politiques immédiates); de ce fait, le problème a un tour intergénérationnel inédit tout en plongeant dans l'urgence du plus court terme qui est celui de la prochaine échéance diplomatique ou de politique intérieure ;

- l'ampleur des incertitudes scientifiques qui demeurent dans les connaissances scientifiques de base, surtout pour la régionalisation des impacts climatiques, mais aussi pour les scénarios de développement économique et technologique à long terme dont dépendent les trajectoires d'émission de GES ; les données scientifiques n'offrent pas de repères naturels évidents sur lesquels l'action de prévention pourrait se caler de façon consensuelle ;

- la mise en cause de quasiment toutes les activités humaines (production, consommation, transports), du fait des émissions de gaz résultant de l'usage de l'énergie fossile, de la production agricole (élevage bovin, riziculture) ou de l'exploitation forestière (déforestation); il ne suffit donc pas de trouver un accord avec quelques grands groupes industriels comme pour la production des CFC, ni de s'en tenir à des substitutions technologiques; on doit considérer des instruments d'action ayant de l'ampleur et on doit se préoccuper des effets d'ensemble sur l'économie et plus seulement d'effets ponctuels ; s'il y a un problème pour lequel il est légitime de raisonner en termes de changement de modes de développement, c'est bien celui-là.

Dans un tel contexte, les raisonnements économiques ne doivent certainement pas être délaissés; ils doivent cependant être remaniés autour d'une configuration appropriée aux univers scientifiquement incertains et controversés (Godard, 1993). 


\section{De l'incertitude aux controverses : le basculement vers une approche séquentielle de la décision}

En univers stabilisé, le principe de rationalité économique est généralement interprété comme l'idée que chaque action possible ou chaque option de choix peut être évaluée à partir d'une comparaison des coûts et des avantages associés à chacune d'entre elles. S'agissant de décisions publiques engageant la collectivité, le principe de 'l'individualisme moral' qui soustend l'analyse de l'économie du bien-être conduit à préconiser la mesure de chaque composante (coût ou avantage) à partir des préférences individuelles des agents économiques concernés. Dans une économie de marché, ces préférences s'expriment usuellement sous la forme de consentements à payer pour accéder aux biens marchands. Quand les marchés sont parfaits et complets, les prix de marché sont supposés exprimer les consentements à payer marginaux qui se dégagent à l'équilibre économique. Lorsque les marchés ne sont pas complets (il n'existe pas de marchés à terme pour tous les biens) ou imparfaits (présence d'effets externes), les prix de marché ne reflètent plus correctement les coûts économiques de l'usage des biens et doivent être corrigés avant d'être utilisés dans les évaluations économiques. C'est ce que font les analyses coûts-avantages qui introduisent un certain nombre de correctifs par rapport aux prix de marché courants.

Cette démarche suscite généralement beaucoup d'espoirs chez les décideurs qui sont demandeurs d'évaluations, du fait des chiffrages qu'elle permet et des conclusions précises auxquelles elle aboutit, même si cette précision est parfois illusoire (Cohen de Lara et Dron, 1997). Cependant, en univers scientifiquement controversé, l'aide directe à la décision qu'il est possible de retirer de cette approche s'est révélée limitée, voire contreproductive. Au sein du GIEC, contrairement aux attentes, l'analyse coûts-avantages n'a pas servi d'élément de formation d'un consensus autour de données objectives, mais bien involontairement d'élément de réactivation des oppositions les plus fondamentales entre les parties en présence, tout particulièrement entre pays industriels et pays en développement. Au cœur des controverses, on trouve quatre questions (Azar, 1998): le traitement des événements catastrophiques à faible probabilité ; l'évaluation des dommages touchant les actifs naturels non marchands et les pertes en vie humaine ; le choix d'un taux d'actualisation en contexte intergénérationnel, ce taux analogue au taux d'intérêt qui est utilisé pour rendre comparables les coûts et les avantages se réalisant à des périodes différentes; les hypothèses de compensation potentielle des dommages entre générations différentes qui sous-tendent certains critères de décision.

Du fait de ces controverses, de l'horizon temporel très long considéré et de l'ampleur des incertitudes, l'élément structurant de la problématique normative de la décision a basculé du cadre d'analyse coûts-avantages vers ce qu'on appelle une approche séquentielle des stratégies d'action. Cette approche séquentielle revient à renoncer à l'idée d'optimiser une fois pour toutes les trajectoires d'émission de long terme, au profit de l'identification du noyau de décisions à prendre à court et moyen terme, en sachant que ces décisions devront être complétées ou révisées à différents horizons. L'attention est centrée sur le choix du meilleur moment d'engagement de l'action (agir aujourd'hui, demain, après-demain) en tenant compte à la fois des constantes de temps respectives des phénomènes physiques, écologiques, économiques et politiques, des perspectives d'apprentissage et d'amélioration des connaissances scientifiques, mais aussi des possibilités attachées au progrès technique. La 
dimension de la réversibilité des engagements, qui doit permettre aux décideurs d'être en position de bénéficier des améliorations futures de l'information, devient un élément important de jugement sur les options d'action.

Les questions 'quand agir ?' et 'avec quelle intensité' se sont alors trouvées placées sous le faisceau de deux types d'argumentations :

- d'un côté, reporter l'action permet de ne pas provoquer une obsolescence accélérée des équipements productifs et de réduire les coûts immédiats d'adaptation; de reporter sur le futur les coûts de l'action en accord avec un critère de préférence pour le présent, une des composantes habituelles de l'actualisation; de n'engager l'action que sur la base de potentialités techniques améliorées qui en réduiront le coût pour des performances égales ; de ne s'engager qu'au vu d'un état amélioré des connaissances scientifiques des phénomènes climatiques et donc de concevoir des actions plus ajustées (réduction de l'espérance du coût d'erreur) (Manne et Richels, 1995);

- d'un autre côté, agir plus tôt permet d'éviter de s'exposer à des ajustements très sévères si la gravité des risques se confirmait et qu'il fallait précipitamment mettre au rebut des équipements productifs et réorganiser les systèmes énergétiques et les systèmes de transports. Il paraît légitime de postuler qu'il existe un plafond implicite à la part des ressources disponibles pour l'investissement que les générations futures accepteront de consacrer au risque climatique. Dans le cadre d'une éthique du développement durable, la génération actuelle peut difficilement s'attribuer le droit de mettre les générations futures dans la situation d'un impossible choix entre une mobilisation exorbitante des capacités d'investissement autour de cette question climatique et la résignation devant un changement climatique dramatique. Enfin, l'engagement immédiat ou prochain dans l'action offre un signal décisif pour l'orientation du progrès technologique et la disponibilité future de techniques plus avantageuses pour résoudre le problème; faute d'une telle action, ces techniques ne se développeront que peu ou pas du tout (Hourcade, 1997).

Le débat principal tourne désormais autour de ces termes. Dans un cas, il n'y aurait pas lieu de s'engager dans des réductions d'émissions avant 2030, mais seulement de poursuivre les programmes de recherche permettant de lever les incertitudes scientifiques pendantes. Dans l'autre cas, une action immédiate de réduction des émissions se justifie, ne serait-ce que pour infléchir les choix d'infrastructures et susciter de façon crédible une réorientation de la recherche technologique dont dépendent les solutions techniques futures. L'incertitude sur la cible ultime à viser (une concentration atmosphérique de GES de 550 ou de 450 ppmv ?) plaide dans le même sens (Ha-Duong et al., 1997).

\section{De l'évaluation des dommages au prix de la précaution}

Agir maintenant, mais jusqu'à quel point? Les ressources à engager dans la prévention doivent a priori être proportionnées aux dommages qu'on veut éviter. Empruntant la voie tracée avec éclat par Nordhaus (1991), plusieurs économistes ont consacré leurs travaux récents à des tentatives d'évaluation des dommages entraînés par divers niveaux de changement du climat. C'est le cas de Cline (1992), puis Fankhauser (1995) ou Tol (1995). Différentes indications chiffrées ressortent des estimations disponibles et permettent de situer 
les ordres de grandeur. Sur l'économie américaine, l'espérance des dommages marchands, pour un doublement de la concentration atmosphérique du $\mathrm{CO}_{2}$, est évaluée par Nordhaus (1991) à 0,25\% du PNB annuellement. En intégrant les dommages non marchands, Cline (1992) aboutit à 1,16\% du PNB, toujours pour les USA. A l'échelle du monde, Fankhauser (1995) aboutit à un dommage annuel de 1,4\% du PNB mondial et Tol à 1,9\%, leurs estimations variant surtout pour les régions des pays du Sud.

Pour utiles qu'elles soient afin d'introduire le débat, ces évaluations ne doivent pas faire illusion et ne peuvent pas être créditées de la même validité que les estimations de dommages bien cernés et observables. Elles sont en effet contingentes à un ensemble important d'hypothèses qui ne sont pas déraisonnables mais qui ne peuvent pas être tenues pour plus assurées que d'autres. On colle alors davantage à la nature de la situation décisionnelle présente en soulignant la dimension de 'risque majeur' des changements climatiques envisagés.

Le concept de 'risque majeur' débouche sur la détermination du consentement à payer collectif vis à vis de la prévention d'un tel risque, très incertain puisque les surprises ne sont pas exclues, potentiellement catastrophique localement ou régionalement, largement nonréparable, dont les conséquences peuvent être à la fois majeures et éloignées dans le temps. Un tel concept a l'avantage de poser explicitement le problème de l'attitude normative qu'il convient d'avoir dans un contexte non complètement réductible aux types de risques maîtrisables sur la base des lois de probabilité liées à la répétition d'un grand nombre d'événements identiques, base de l'assurance.

Ce type de risque relève d'une culture de précaution et ce n'est pas un hasard si la Convention sur le climat tout à la fois intègre en son article 3.3. l'énoncé du principe de précaution $^{1}$ et pose l'obligation des parties signataires «de prendre des mesures de précaution pour prévoir, prévenir ou atténuer les causes des changements climatiques et en limiter les effets néfastes ». Avec cette référence à la précaution, il s'agit notamment de prendre en compte l'aversion particulière aux risques collectifs imputables aux décisions d'institutions publiques ou de firmes qui est couramment manifestée par les populations. Du point de vue de la théorie économique, les valeurs d'option (en statique), et les quasi-valeurs d'option (en contexte d'incertitude avec irréversibilité et perspective d'amélioration de l'information) doivent au minimum se surajouter à l'espérance mathématique de la valeur des dommages attendus, de même que l'incertitude sur la croissance économique future tend à se traduire par une baisse sensible du taux d'actualisation de référence (Gollier, 1998).

Il est légitime de se demander quel pourrait être, aujourd'hui et pour les prochaines années, le 'prix de la précaution', c'est à dire le consentement collectif à payer pour obtenir un niveau de réduction des émissions de GES suffisant pour ramener le risque à un niveau jugé acceptable. On peut donner quelques jalons à partir d'évaluations économiques disponibles dans la littérature.

\footnotetext{
1.- Cet article le caractérise ainsi : «quand il y a risque de perturbations graves ou irréversibles, l'absence de certitude scientifique absolue ne doit pas servir de prétexte pour différer l'adoption de telles mesures, étant donné que les politiques et mesures qu'appellent les changements climatiques requièrent un bon rapport coût/efficacité, de manière à garantir des avantages globaux au coût le plus bas possible. »
} 


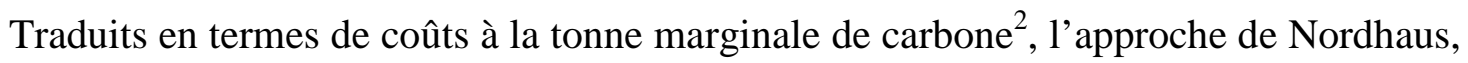
qui ignore la dimension d'incertitude attachée au problème et repose sur une actualisation standard, conduit à une valeur actuelle d'environ 40 FF la tonne, généralement considérée comme une évaluation plancher. Hope et Maul (1996) montrent comment une évaluation de cet ordre peut, par réarrangements successifs des hypothèses visant à prendre en compte l'incertitude et à rendre l'approche comparable avec celle adoptée dans d'autres domaines de risques technologiques ${ }^{3}$, conduit à une valeur moyenne de $140 \mathrm{FF}$ la tonne dans le cadre du modèle PAGE. Cependant, le plafond de la fourchette obtenue avec ce modèle se situe à $300 \mathrm{FF}$, valeur que les auteurs proposent d'adopter comme valeur de référence de la précaution.

Sur une période d'un siècle ou plus, toute évaluation est très sensible à la valeur adoptée pour le taux d'actualisation. Par exemple, selon que l'on retienne $2 \%$ ou $5 \%$ sur cent ans, la valeur actuelle des dommages se réalisant à cet horizon se situe déjà dans un rapport de 1 à 20. Cependant, les simulations réalisées par Manne et Richels (1995) montrent que le choix de se caler sur un scénario mondial dans lequel les concentrations atmosphériques de $\mathrm{CO}_{2}$ ne dépasseraient pas $550 \mathrm{ppm}$ à long terme pourrait représenter une 'meilleure' option robuste face aux incertitudes sur le choix du taux d'actualisation entre 2 et $5 \%$. Or, d'après le GIEC, pour parvenir à ce résultat, la moyenne annuelle globale des émissions ne devra pas dépasser la moyenne actuelle au cours du siècle prochain et devra même devenir nettement inférieure avant la fin du XXIième siècle et au-delà (Bruce et al., 1997) : tout dépassement du niveau actuel d'émissions devrait donc être compensé par une baisse des émissions futures en dessous du niveau actuel, en moyenne mondiale. ${ }^{4}$

Les mêmes auteurs donnent une fourchette de valeur pour la tonne de carbone évitée selon les scénarios. Dans le scénario central, sans surprise, cette valeur commence à $35 \mathrm{FF}$ en 2000 pour parvenir à $60 \mathrm{FF}$ en 2020 . Dans un scénario combinant un changement climatique drastique (élévation de la température moyenne de $5^{\circ} \mathrm{C}$ pour un doublement de la concentration atmosphérique en GES) et un consentement à payer élevé (calibrage à $2 \%$ du revenu pour une augmentation de température de $1^{\circ} \mathrm{C}$ ), la valeur de la tonne de carbone évitée est de $680 \mathrm{FF}$ en 2000, pour dépasser les $1200 \mathrm{FF}$ avant 2020. La valeur de la précaution se situe pour eux dans cette large fourchette (entre 35 et $680 \mathrm{FF}$ en 2000 et entre 60 et $1200 \mathrm{FF}$ en 2020), sa place exacte dépendant des probabilités subjectives accordées par les décideurs aux différents scénarios. L'objectif de stabilisation durable des émissions mondiales à leur niveau de 1990, sans dépassement aucun, ne pourrait se justifier aux yeux de ces auteurs que dans une logique d'évitement du scénario le moins favorable pris en compte, qui est aussi un scénario qui leur paraît avoir une faible probabilité. Le document de synthèse du GIEC note cependant que : «dans le cas précis d'une stabilisation des émissions au niveau de 1990, la plupart des études aboutissent à une estimation du coût annuel comprise entre -0,5\% du PIB (ce qui équivaudrait à un gain total d'environ 60 milliards de dollars pour les pays de l'OCDE, au niveau actuel du PIB) et $+2 \%$ du PIB (ce qui équivaudrait à une perte d'environ 240 milliards de dollars) pendant les prochaines décennies» (Bruce et al., 1997, p. 518).

\footnotetext{
2.- 1 tonne de carbone donne 3,66 tonnes de $\mathrm{CO}_{2}$. Une taxe de $60 \mathrm{FF}$ la tonne de carbone revient donc à une taxe de 16 FF la tonne de $\mathrm{CO}_{2}$.

${ }^{3}$.- Notamment en prenant une valeur unique pour la vie humaine statistique, quelle que soit la région du globe, comme on le fait semble-t-il dans le nucléaire.

4.- Cette moyenne mondiale s'élève à $1,1 \mathrm{t}$. de carbone, répartie entre une valeur moyenne de $2,8 \mathrm{tC}$ pour le groupe des pays de l'OCDE et des pays Est-européens en transition, et 0,5 tC dans les pays en développement.
} 
Sur la base de ces différentes évaluations encore très dispersées, on peut raisonnablement défendre un prix actuel de la précaution à l'échelle mondiale entre 300 et $600 \mathrm{FF}$ la tonne de carbone évitée. Une telle évaluation ne prend en compte que les risques climatiques et ignore les avantages secondaires qui pourraient résulter d'une politique de réduction des émissions des GES. Compte tenu des responsabilités propres qui incombent aux pays industriels ${ }^{5}$ et de leurs revenus par habitant plus élevés que la moyenne mondiale, le prix de la précaution à payer dans ces pays aurait a priori à être plus élevé que le prix mondial.

\section{Actions sans regrets et double dividende fiscal}

Lorsqu'on réduit les émissions de $\mathrm{CO}_{2}$ et d'autres gaz à effet de serre, lorsqu'on économise sur la consommation d'énergie fossile, on réduit en même temps un ensemble d'autres « effets externes » négatifs, notamment ceux liés aux dommages de proximité de la pollution de l'air par d'autres gaz issus de la combustion énergétique : $\mathrm{SO}_{2}, \mathrm{CO}, \mathrm{NOx}, \mathrm{COV}$, particules, etc. Il se trouve aussi que ces opérations peuvent servir de levier à l'introduction de technologies moins coûteuses, à l'usage, que les techniques en place.

La prise en compte de ces potentiels d'action dits «sans regrets » puisqu'à coût net négatif ou nul, permet de rendre la précaution moins coûteuse à assumer et déplace le calendrier optimal de l'action en faveur d'une action plus précoce. Il reste à trouver les politiques adaptées à la mobilisation efficace de ces actions «sans regrets ». Une des composantes à privilégier est le recours à des instruments économiques qui, outre leur propriétés d'incitation, de souplesse et d'efficacité économique dans la répartition des actions, permettront d'un côté d'adresser un signal-prix pour orienter le progrès technique à moyen et long terme et d'un autre côté d'engendrer ce qu'on appelle un double dividende (Bureau et Hourcade, 1998).

Il y a en effet un deuxième élément, tout aussi important, de transformation de la problématique de l'action de prévention des risques climatiques, d'ailleurs susceptible d'affecter à la fois son calendrier, ses moyens et son intensité : le deuxième dividende fiscal qui peut être obtenu lorsque les instruments de politiques choisis fournissent l'opportunité d'un redéploiement de la fiscalité générale. L'incorporation de nouvelles ressources fiscales, comme celles qui résulteraient d'une taxe sur le carbone ou de permis négociables vendus aux enchères par les autorités publiques, permettrait de réduire ou supprimer d'autres impôts ou charges hérités de l'histoire mais devenus la source de distorsions ou de déséquilibres économiques. Ce deuxième dividende est ainsi à la mesure des imperfections de l'organisation actuelle des prélèvements obligatoires qui altèrent les incitations à l'embauche, au travail, à l'épargne, à l'innovation, à la consommation de tel bien plutôt que de tel autre, etc., mais aussi à la mesure de la capacité collective de la société à y remédier. C'est sur l'ampleur de ce double dividende, et sur sa capacité à plus que compenser ou seulement atténuer les coûts directs des réductions d'émission, que porte aujourd'hui le débat entre économistes (courbes A2 ou A3 sur la Figure 1).

Parmi les distorsions économiques les plus lourdes attachées aux institutions qui ont été mises en place depuis l'après-guerre, il faut compter l'effet pervers sur la longue durée du

\footnotetext{
5.- La Convention sur le climat fait état de « responsabilités communes mais différenciées » et souligne la responsabilité première des pays industriels.
} 
choix d'asseoir la sécurité sociale sur les salaires, provoquant ainsi une augmentation artificielle du coût du travail pour les employeurs. Cela a introduit une incitation accrue à recourir à des technologies intensives en capital mais économisant le travail, même là où ce type de solution ne s'imposait pas comme une condition de l'accès à la technologie moderne. Cela a aussi renforcé les freins à l'embauche lorsque la conjoncture et les perspectives de marché étaient incertaines et fluctuantes ${ }^{6}$. C'est cette distorsion involontaire des choix économiques et techniques que l'on pourrait contribuer à réparer en s'appuyant sur la politique de l'effet de serre. Cela serait possible en poursuivant le transfert de l'assiette des charges sociales qui pèsent aujourd'hui sur les salaires, en particulier pour le travail peu qualifié.

Outre ses avantages économiques, la prise en compte des potentiels 'd'action sans regrets' et 'du double dividende fiscal' permettrait d'enraciner une politique visant des objectifs à très long terme dans la solution de problèmes d'aujourd'hui. En revanche, des politiques de l'effet de serre mal conçues, mais proches des façons de faire traditionnelles en Europe, à base de réglementations contraignantes et de normes uniformes, pourraient se révéler inutilement très coûteuses, au point de remettre en cause le bien-fondé d'une politique de réduction immédiate des émissions comme celle qui a été engagée à Kyoto.

Coût marginal de la tonne de carbone évitée

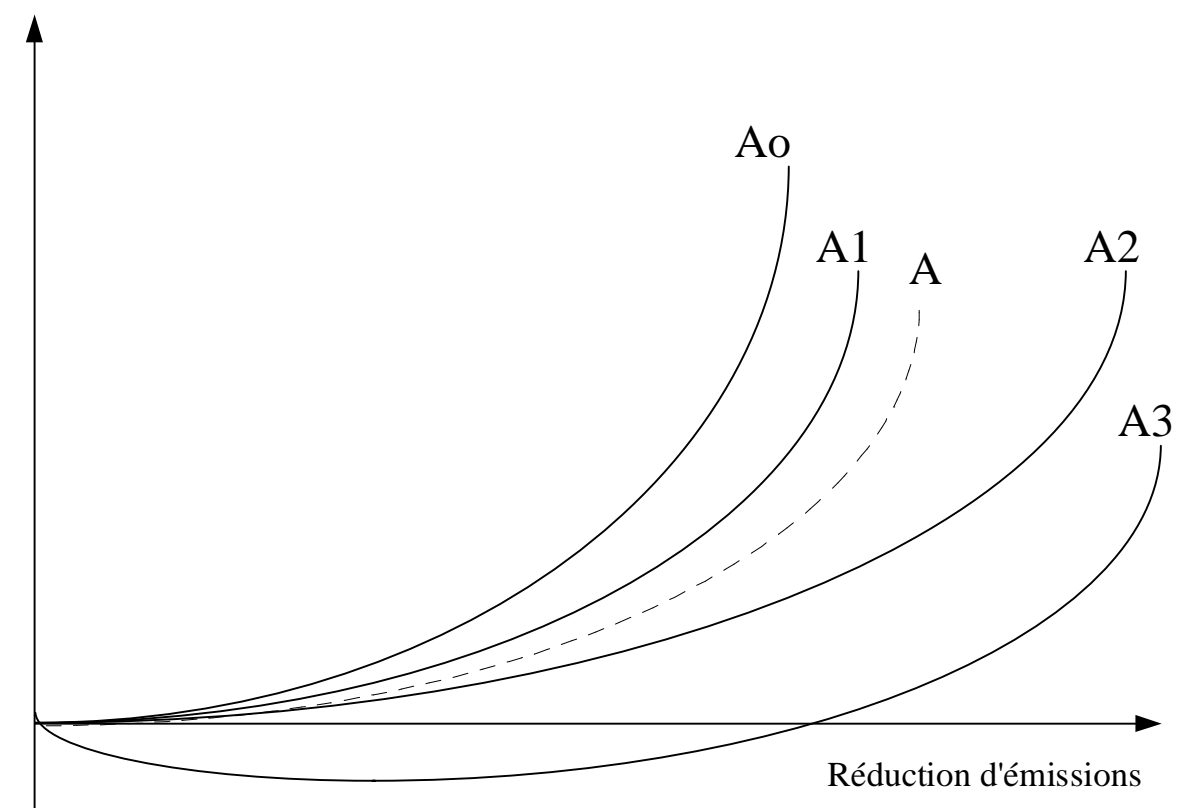

Figure 1: les coûts d'une taxe sur le carbone

adaptée d'après Bureau et Hourcade (1998) et Bruce et al. (1997)

A coût marginal sectoriel des techniques de réduction des émissions

$\mathrm{A}_{0} \quad$ coût macroéconomique marginal sans recyclage du produit de la taxe

A1 coût macroéconomique marginal avec recyclage proportionnel du produit de la taxe

A2 coût macroéconomique marginal avec recyclage du produit de la taxe focalisé sur les impôts et charges existants les plus distorsifs - option 1 .

\footnotetext{
${ }^{6}$.- Plus les charges sont élevées, pour un même salaire net, plus les coûts d'une erreur d'appréciation sur les débouchés sont élevés et donc dissuasifs pour les employeurs ayant plutôt une aversion à ce type de risque.
} 
A3 coût macroéconomique marginal avec recyclage du produit de la taxe focalisé sur les impôts et charges existants les plus distorsifs - option 2.

\section{Le Protocole de Kyoto ou la consécration des permis négociables}

Les émissions de $\mathrm{CO}_{2}$ sont en passe de devenir un critère stratégique majeur pour les politiques énergétiques des pays industriels. Pour ceux de ces pays qui sont réunis dans l'Annexe 1 de la Convention, le Protocole négocié à Kyoto prévoit des objectifs quantifiés spécifiques à chacun et destinés à être juridiquement contraignants, pour l'émission de six gaz durant la période 2008-2012 : $\mathrm{CO}_{2}, \mathrm{CH}_{4}, \mathrm{~N}_{2} \mathrm{O}$ et trois composés fluorés. Les pays de l'Union européenne ont reçu chacun un quota annuel moyen de 92\% des émissions réalisées en 1990, les États-Unis de 93\%, le Japon de 94\%, la Russie de 100\%, l'Australie de 108\%, pour ne citer que les principaux pays émetteurs. Le rationnement quantitatif des émissions de ces GES et en particulier du $\mathrm{CO}_{2}$ revient à rationner l'usage de l'énergie fossile de différentes sources (charbon, pétrole, gaz) dans ses différentes formes d'emploi (carburant automobile, chauffage de bâtiments, production d'électricité, processus industriels, etc.).

Cependant, le même Protocole a admis dans leur principe différents mécanismes de flexibilité et notamment la possibilité pour les États ayant souscrit à de tels objectifs quantifiés (OCDE, Russie et autres pays d'Europe de l'Est) d'échanger leurs quotas entre eux. Les pouvoirs publics, en France et dans les autres pays, auront donc à arbitrer entre le taux d'effort à réaliser sur le territoire national et le degré de recours aux mécanismes de flexibilité et d'échange international de quotas d'émission.

Quels sont donc les mécanismes de flexibilité prévus ? Ils sont au nombre de cinq et appartiennent à la famille des permis négociables (emissions trading), prise au sens large.

\subsection{Ce que sont les mécanismes de flexibilité adoptés à Kyoto}

\section{Le commerce intergouvernemental des quotas d'émissions entre pays de l'Annexe 1}

L'article 17 prévoit la possibilité pour ces pays d'échanger leurs quotas d'émission en vue d'honorer leurs obligations pour la période 2008-2012. Cette possibilité ne peut être utilisée que de façon complémentaire aux mesures intérieures prises par ces pays. Les modalités de ce commerce intergouvernemental seront définies lors de la prochaine conférence à Buenos Aires en novembre 1998.

\section{Les crédits d'émissions au niveau de projets dans les pays de l'Annexe 1}

L'article 6 prévoit la possibilité pour les États de l'Annexe 1 de transférer entre eux des unités de réduction des émissions qui résulteraient de la réalisation sur le territoire de l'un d'eux de projets financés par un autre. Ces transferts viendraient s'ajouter ou se soustraire aux quotas de chaque pays. Les projets en question doivent viser soit la réduction des émissions, soit le retrait des gaz présents dans l'atmosphère au moyen de «puits à carbone » (sinks) comme les forêts et les sols. Cette possibilité ne peut être utilisée que si ces projets permettent des réductions supplémentaires s'ajoutant à celles qui auraient été réalisées en l'absence de ces projets. Chaque État peut, sous sa responsabilité, autoriser d'autres personnes morales, 
comme des entreprises ou des $\mathrm{ONG}$, à participer à ces actions et à bénéficier des crédits d'émission.

\section{Le mécanisme de développement propre (MDP)}

L'article 12 prévoit la création d'un «mécanisme de développement propre » entre pays en développement (hors Annexe 1) et pays industriels (Annexe 1). Des projets réalisés sur le territoire des premiers pourront obtenir des réductions d'émissions certifiées qui pourront d'un commun accord, bien que les pays en développement n'aient pris aucun engagement quantifié, se transformer en crédits d'émission transférables aux États industriels. Ces crédits seront alors comptabilisés par ces États pour satisfaire leurs obligations. Les réductions certifiées ainsi obtenues entre les années 2000 et 2007 pourront être utilisées pour les obligations de la période 2008-2012. Pour être créditées, les actions menées devront avoir des « effets réels, mesurables et à long terme du point de vue du changement climatique » et créer des réductions supplémentaires par rapport à celles qui se seraient produites en l'absence de ces projets. L'organisation de ce mécanisme se fera sur une base multilatérale.

\section{La mise en ouvre conjointe au sein de bulles internationales, pour les pays de l'Annexe 1}

D'après l'article 4, les États de l'Annexe 1 peuvent décider de satisfaire de façon conjointe les obligations quantifiées qui leur incombent pour la période 2008-2012 en établissant un accord séparé qui préciserait de nouveaux objectifs d'émissions pour chaque Partie de cet accord. Le montant agrégé des émissions d'une telle «bulle» ne peut pas être supérieur au total des engagements individuels qui ont été souscrits à Kyoto par les États participants. L'Union européenne était particulièrement intéressée par cette disposition et a déjà procédé à une nouvelle répartition interne des obligations entre ses États-membres dans laquelle la France a reçu un quota de $100 \%$ de ses émissions de 1990.

\section{La mise en réserve pour des périodes ultérieures (États de l'Annexe 1)}

Les États de l'Annexe 1 ont la possibilité reconnue par l'article 3.13 de mettre en réserve pour une période d'engagement ultérieure les quotas d'émission qui demeureraient inutilisés pendant la période 2008-2012.

\subsection{La possibilité d'échange, un facteur important de réduction des coûts de la politique de l'effet de serre}

Les possibilités d'échange sont intéressantes à plusieurs titres : flexibilité dans le choix de politiques par chaque pays, en particulier dans le domaine énergétique ; association des pays en développement pour le Mécanisme de développement propre. Du fait des différences importantes dans les coûts marginaux de réduction des émissions d'une région du globe à l'autre, elles sont aussi un facteur important de réduction des coûts totaux supportés par l'économie mondiale pour la réalisation d'un niveau donné de réduction des émissions. Par exemple, en partant d'une évaluation moyenne de coût annuel de contrôle des émissions de $1 \%$ du PIB, et d'un PIB français se situant autour de 8000 GF en 2010, une économie de coûts d'un facteur deux ou trois, comme celle que pourrait procurer la flexibilité la plus large, pourrait déboucher sur un gain annuel de 40 à 50 GF pour la France. Certes, la totalité de ce gain ne sera pas à portée de main, car il faudra que le pays engage de toute façon des politiques à plus long terme de réduction des émissions à partir du territoire national. La 
moitié seulement serait déjà un gain très appréciable qui pourrait être affecté à d'autres priorités de la collectivité.

Le mécanisme d'abaissement des coûts à travers l'échange de permis d'émission est illustré sur la figure 2 qui représente les fonctions de coût marginal (coût de réduction d'une unité supplémentaire d'émission, qui est la fonction dérivée de la fonction de coût total de réduction des émissions) de deux pays 1 et 2 et les gains d'un échange entre eux.

Le même raisonnement est facilement transposable entre entreprises de secteurs ou pays différents.

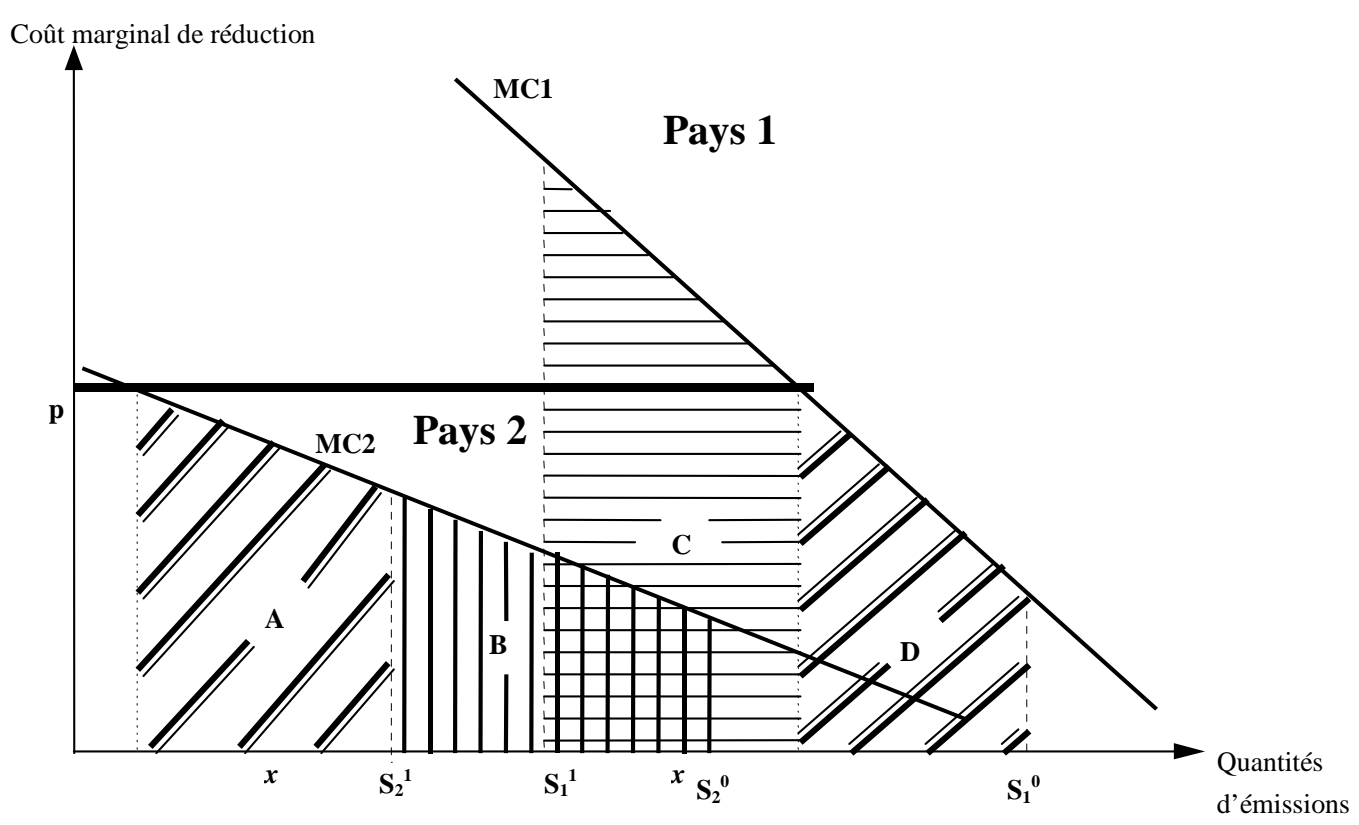

Figure 2: Une réduction des émissions de $50 \%$

Dans cette figure, on représente la position de deux pays $S_{1}$ et $S_{2}$, dont les niveaux d'émission respectifs avant un engagement de réduction sont $S_{1}{ }^{0}$ et $S_{2}{ }^{0}$. Avec un objectif uniforme de réduction de $50 \%$ des émissions, $S_{1}$ doit supporter un coût total représenté par l'aire $C+D$, de façon à atteindre le niveau $S_{1}{ }^{1}$ et $S_{2}$ un coût représenté par l'aire B pour atteindre le niveau $S_{2}{ }^{1}$. Ces niveaux de coûts sont certes très inégaux dans l'absolu; l'équilibre imposé $\left(\mathrm{S}_{1}{ }^{1}, \mathrm{~S}_{2}{ }^{1}\right)$ correspond surtout à des coûts marginaux sensiblement différents. On voit bien que, pour les intéressés comme pour la communauté internationale prise dans son ensemble, la réalisation d'une nouvelle allocation des efforts induirait une économie de coûts tout en satisfaisant une même contrainte quantitative d'ensemble. C'est ce que permet un mécanisme d'échange de quotas entre les deux pays, s'équilibrant au prix $\boldsymbol{p}$. En accroissant ses réductions d'émission de $\boldsymbol{x}$ unités, $\mathrm{S}_{2}$ dégage un surplus de droits à émettre qu'il peut revendre à $S_{1}$ qui limite d'autant son effort de réduction. $\mathrm{S}_{1}$ supporte désormais un coût net égal à $\mathrm{D}+\boldsymbol{p} \boldsymbol{x}$ et $\mathrm{S}_{2}$ un coût net de $\mathrm{B}+\mathrm{A}-\boldsymbol{p} \boldsymbol{x}$. Le coût total $\mathrm{B}+\mathrm{D}+\mathrm{C}$ est ramené à $\mathrm{B}+\mathrm{D}+\mathrm{A}$, avec $\mathrm{A}<\mathrm{C}$.

Dans une perspective dynamique, les écarts de coûts entre pays vont dépendre de la répartition des objectifs quantifiés qui sera adoptée pour les périodes postérieures à 2012. Si cette répartition obéit principalement à des considérations d'équité, elle correspondra à une 
allocation économiquement inefficace et justifiera de façon durable et pas seulement transitoire le recours aux mécanismes d'échange.

\subsection{Opportunités, dangers et enjeux stratégiques pour la France}

Le recours aux échanges non entravés des permis négociables représente à la fois un enjeu économique et politique important mais aussi certains dangers qu'il convient d'expliciter si l'on veut cerner les enjeux des négociations à venir.

\section{Viser la restauration de marges de manœuvre stratégique dans le domaine de la production électrique}

Certains pays au sein de l'Union européenne, y compris la France à travers certains de ses négociateurs, ont cherché depuis la Conférence de Kyoto à réduire au maximum la portée des instruments de flexibilité instaurés par cette conférence. Or, la combinaison de la fixation d'objectifs rigides par pays, de la référence aux émissions de 1990 pour les déterminer, et d'une différenciation des efforts donnant une prime aux 'gros pollueurs', comme celle qui fut scellée à Kyoto, représenterait le plus mauvais scénario pour la France ! Il se traduirait par des coûts élevés et une rigidité stratégique forte, alors même que notre pays compte parmi les pays industriels qui émettent le moins de $\mathrm{CO}_{2}$ par habitant. Les conséquences en seraient défavorables pour la compétitivité économique du pays, mais aussi pour l'exercice de la démocratie à propos des choix technologiques.

En effet si la communauté internationale s'en tenait, au delà de 2012, à l'approche rigide qui vient d'être esquissée, et si la France continuait à se voir attribuer des quotas d'émission de GES non croissants par rapport à 1990, cette dernière ne pourrait plus modifier sa politique énergétique dans un sens impliquant davantage d'émissions de GES. Le nucléaire représente aujourd'hui près de $80 \%$ de la production électrique et on ne voit pas comment la croissance des émissions du secteur des transports routiers pourrait être évitée (voir le tableau 1): même impressionnants, les gains unitaires sur les performances des véhicules continueront à l'avenir d'être largement neutralisés par l'augmentation du trafic, la montée en gamme de puissance de la moyenne des véhicules du parc et l'extension d'équipements annexes consommateurs d'énergie comme la climatisation, sans parler des inerties du renouvellement du parc automobile. A l'heure du remplacement du parc électronucléaire existant, en l'état des technologies de base et des modes de vie, et en dépit des actions visant à améliorer l'efficacité énergétique, nécessaires pour réduire les émissions par rapport à l'évolution tendancielle, la France ne pourrait pas renoncer à l'option nucléaire ou même envisager de diversifier de façon sensible son parc de production en recourant par exemple aux cycles combinés à gaz. C'est ce qui ressort nettement des travaux du Groupe Énergie 2020 du Commissariat général du Plan, en particulier des trois scénarios énergétiques qui ont été élaborés pour la France (CGP, 1998). Le parc nucléaire représenterait en effet à l'horizon 2020 une économie annuelle sur les émissions de $\mathrm{CO}_{2}$ d'environ $30 \mathrm{MtC}$, soit $29 \%$ des émissions de 1990 (voir le tableau 1). Pour dénucléariser son appareil de production électrique, la France aurait donc besoin d'une marge de flexibilité d'au moins $30 \%$ de ses émissions de 1990. Faute d'en disposer, elle ne pourrait retrouver une autonomie stratégique qu'à beaucoup plus long terme, cinquante ans plus tard, lorsque des ruptures technologiques 
majeures (énergie de biomasse ? généralisation des véhicules électriques ?...) se seraient produites et que les nouvelles centrales mises en place viendraient elle-même au bout de leur vie active.

\section{Tableau 1}

\section{Prospective des émissions de $\mathrm{CO}_{2}$ d'origine énergétique en France aux horizons 2010 et 2020}

\begin{tabular}{|c|c|c|c|c|c|c|c|}
\hline Année & \begin{tabular}{|l} 
Secteurs \\
en \% sauf total
\end{tabular} & \multicolumn{6}{|c|}{ Emissions de $\mathrm{CO}_{2}$} \\
\hline \multirow[t]{4}{*}{1990} & Industrie & \multicolumn{6}{|c|}{$30,8 \%$} \\
\hline & Résid./tertiaire & \multicolumn{6}{|c|}{$28,7 \%$} \\
\hline & Transports & \multicolumn{6}{|c|}{$38 \%$} \\
\hline & Total $(\mathrm{MtC})$ & \multicolumn{6}{|c|}{104,5} \\
\hline \multirow[t]{5}{*}{2010} & Scénarios & \multicolumn{2}{|c|}{$\begin{array}{l}\text { Société de } \\
\text { marché (S1) }\end{array}$} & \multicolumn{2}{|c|}{$\begin{array}{l}\text { Etat industriel } \\
(\mathrm{S} 2)\end{array}$} & \multicolumn{2}{|c|}{$\begin{array}{c}\text { Etat protecteur de } \\
\text { l'environnement }(\mathrm{S3})\end{array}$} \\
\hline & Industrie & \multicolumn{2}{|c|}{$25,4 \%$} & \multicolumn{2}{|c|}{$24,9 \%$} & \multicolumn{2}{|c|}{$21,2 \%$} \\
\hline & Résid./tertiaire & \multicolumn{2}{|c|}{$28,7 \%$} & \multicolumn{2}{|c|}{$27,5 \%$} & \multicolumn{2}{|c|}{$30,6 \%$} \\
\hline & Transports & \multicolumn{2}{|c|}{$43,9 \%$} & \multicolumn{2}{|c|}{$45,4 \%$} & \multicolumn{2}{|c|}{$45,6 \%$} \\
\hline & Total $(\mathrm{MtC})$ & \multicolumn{2}{|c|}{131,1} & \multicolumn{2}{|c|}{119} & \multicolumn{2}{|c|}{101,4} \\
\hline \multirow[t]{5}{*}{2020} & & $V 40$ & V30 & $V 40$ & $V 30$ & $V 40$ & V30 \\
\hline & Industrie & $23,2 \%$ & $24,6 \%$ & $23,2 \%$ & $23,1 \%$ & $21,8 \%$ & $23,3 \%$ \\
\hline & Résid./tertiaire & $28,8 \%$ & $35,5 \%$ & $27,4 \%$ & $27,6 \%$ & $29,6 \%$ & $30,1 \%$ \\
\hline & Transports & $46,1 \%$ & $38,4 \%$ & $47,3 \%$ & $47,2 \%$ & $46,1 \%$ & $44,1 \%$ \\
\hline & Total $(\mathrm{MtC})$ & 150,2 & 180,7 & 131,5 & 131,8 & 106,1 & 111,0 \\
\hline
\end{tabular}

Source : Commissariat général du plan (1998)

Total des émissions directes et indirectes, après imputation des émissions de la production primaire au prorata des consommations d'électricité et de produits pétroliers raffinés

Les scénarios «Société de marché - S1 », «État industriel - S2 », «État protecteur de l'environnement -S3 » correspondent à trois évolutions possibles de la société française à l'intérieur desquelles les évolutions et choix énergétiques prennent place. Les variantes V40 et V30 correspondent aux deux hypothèses de durée de vie des centrales nucléaires du parc existant : quarante ou trente ans. Dans le deuxième cas, c'est à partir de 2007 que le problème du remplacement se posera, tandis que dans le premier cas, l'échéance se situe à partir de 2017. Dans S1-V30 les centrales nucléaires sont remplacées par des turbines à gaz à cycle combiné, même pour la production d'électricité en base, d'où une forte croissance des émissions de CO2. Seul le scénario S3, tout à fait non tendanciel, apparaît compatible avec une stabilisation en 2010 des émissions de $\mathrm{CO} 2$ sur le seul territoire français. Il incorpore dans V30 une hypothèse d'un nouvel équipement nucléaire de type EPR pour $30 \mathrm{GW}$, ce qui fait que dans ce scénario «environnemental », le nucléaire assure encore $60 \%$ de la production électrique ; avec V40, l'équipement actuel suffit à satisfaire la demande sans investissements de remplacement.

Si le scénario de la rigidité du régime climatique devait l'emporter, à partir de 20102015, les centrales nucléaires existantes devraient être remplacées par d'autres centrales nucléaires, quel que soit le gouvernement en place, sans que les citoyens puissent peser sur un 
engagement de cette nature qui lierait leur destinée à la technologie nucléaire pour une durée d'au moins quarante années supplémentaires. Quel que soit le jugement que chacun portera, à l'heure des décisions, sur les avantages et les inconvénients relatifs, vis à vis d'autres énergies, d'une filière nucléaire qui aura bénéficié de progrès techniques par rapport aux équipements antérieurs, ce déni de choix pourrait avoir des conséquences politiques inquiétantes dans une période de vive sensibilité de l'opinion envers la manière dont les institutions gèrent les risques collectifs (Godard, 1997a).

Par ailleurs, sans la flexibilité apportée par le commerce des permis, toute erreur de prévision dans le domaine de l'évolution des trafics routiers et des consommations énergétiques des transports devrait être payée par les secteurs résidentiel (chauffage) et surtout industriels.

Si l'on s'en tient aux éléments rationnels du dossier, la France compte parmi les premiers pays à avoir besoin de flexibilité dans le choix des moyens d'honorer les obligations qu'elle a contractées dans le cadre de la Convention sur le climat. Au vu des solutions aujourd'hui discutées, le commerce de permis d'émission procure cette flexibilité. Refuser ou entraver cet instrument empêcherait notre pays de recouvrer la maîtrise démocratique de ses choix énergétiques futurs, lui imposerait inutilement et de façon récurrente des coûts élevés, et pourrait lui imposer une gestion difficile d'un éventuel manque de maîtrise de la croissance des transports routiers.

\section{Rechercher une harmonisation internationale suffisante des règles d'organisation des permis négociables}

Les bonnes performances économiques susceptibles d'être apportées par l'échange de permis d'émission dépendent de la solidité du cadre institutionnel et de la crédibilité des règles ayant à assurer l'organisation concurrentielle des échanges ${ }^{7}$. Or, parmi les craintes exprimées, certaines touchent aux risques que n'apparaissent d'importantes distorsions de la concurrence sur les marchés de produits industriels si chaque gouvernement peut procéder comme il l'entend pour organiser des permis négociables sur son territoire, alors même que ces permis pourront ensuite faire l'objet d'échanges internationaux. Ainsi, tel pays pourrait exonérer tel secteur pour lui attribuer un avantage compétitif et reporter l'essentiel de l'effort sur tel autre. Un autre État pourrait acquérir des permis sur le marché international et les remettre gratuitement à la disposition de certaines firmes particulièrement engagées dans la compétition internationale, etc. L'évolution du droit de la concurrence au niveau européen et international n'a eu de cesse de chercher à préserver les échanges économiques de telles manipulations. Il serait paradoxal que la Convention sur le climat soit l'occasion d'ouvrir la porte en grand à de telles pratiques. Pour l'éviter, les États doivent s'accorder sur un minimum de règles communes. C'est pourtant ce type d'harmonisation que des pays comme les États-Unis ont constamment rejeté depuis 1992 au nom du respect du principe de souveraineté des États.

Deux remarques s'imposent cependant. Les effets distorsifs pour l'économie risquent d'être d'autant plus importants que les structures de marchés sont éloignées d'un

\footnotetext{
${ }^{7}$ C'est l'une des principales leçons de l'expérience américaine de permis négociables pour les émissions de SO2 ; voir Godard (1997b) et Godard et Henry (1998).
} 
fonctionnement concurrentiel 'parfait' et reposent sur l'activité d'un nombre limité de firmes en position d'oligopoles. Il serait donc souhaitable de viser une organisation des permis négociables qui soit la plus ouverte et la plus concurrentielle possible. Par ailleurs, les risques les plus élevés de distorsion économique seraient le fait d'un système de zonage et de quotas non échangeables qui imposerait aux pays de façon rigide des obligations asymétriques entre les pays de l'Annexe 1 et les pays en développement, comme entre pays de l'Annexe 1. Limiter les échanges de permis n'est donc pas une parade pour se prémunir contre les actions stratégiques éventuelles d'autres pays. Le problème de l'harmonisation des politiques nationales de l'effet de serre se pose même sans permis négociables (Godard, 1997b).

Il y a un autre aspect. Un État qui déciderait de distribuer gratuitement des permis à celles de ses entreprises les plus exposées à la concurrence internationale n'obligerait-il pas les autres à en faire de même, afin de ne pas désavantager ses propres entreprises, détruisant ainsi toute possibilité réelle de les impliquer dans une réforme fiscale écologique ? Si c'était le cas, et c'est vraisemblable, c'est la possibilité de mobiliser le double dividende fiscal qui s'en trouverait limitée ? En effet la logique du double dividende nécessite l'introduction d'une nouvelle fiscalité écologique afin de disposer des ressources fiscales permettant de réduire d'autres impôts et charges plus distorsifs pour l'économie. Seules des taxes d'un montant significatif ou des permis négociables vendus aux agents économiques le permettent. Il serait paradoxal que l'introduction de permis négociables, motivée par la recherche de l'efficacité économique, empêche de mettre en œuvre des réformes fiscales dont on peut attendre un gain économique et social au moins aussi important! Certes les gouvernements pourraient chercher à composer en concevant des dispositifs dérogatoires ou compensatoires pour les industries exposées à la concurrence internationale. De telles activités sont cependant de plus en plus nombreuses et les gouvernements prendraient alors un risque politique nouveau, celui que, sous la pression des intérêts sectoriels, ils soient amenés à étendre exonérations ou compensations de proche en proche à tous les secteurs disposant de lobbies bien organisés et ils ne manquent pas.

Les craintes d'une paralysie des initiatives gouvernementales sur le terrain de la fiscalité écologique sont d'autant plus fondées que c'est ce genre de considérations qui a empêché jusqu'à présent l'aboutissement du projet européen de création d'une taxe sur le carbone, formulé dès $1991^{8}$, et que, pour certains milieux industriels et financiers, l'instrument des permis négociables est vu d'abord comme le moyen d'obtenir le démantèlement des écotaxes existantes ou de faciliter l'appropriation privative des rentes de rareté engendrées par le rationnement des émissions de GES.

La réalisation d'un accord au sein du groupe des pays industriels pour définir des conditions de mise en œuvre équivalentes des permis négociables, ou d'autres instruments, dans les branches industrielles grandes consommatrices d'énergie les plus engagées dans la compétition internationale serait certainement de nature à lever une partie de l'hypothèque que font peser sur la Convention les menaces d'altération de la compétitivité et la crainte des distorsions de concurrence.

Vis à vis des pays en développement qui ne sont pas membres de l'Annexe 1, c'est le Mécanisme de Développement Propre (MDP) qui, bien conçu, permettrait à moyen et long terme d'atténuer de façon importante l'incitation à la délocalisation que représente l'absence

\footnotetext{
${ }^{8}$ Voir une analyse de ce projet dans l'ouvrage du Commissariat général du plan (1993).
} 
d'objectifs chiffrés de limitation des émissions de GES pour ces pays, même si le premier effet à court terme pourrait être plutôt opposé. Si les crédits gagnés dans ces pays ne sont pas reversés entièrement aux pays investisseurs et que le mécanisme est organisé de manière à être très largement accessible aux entrepreneurs des pays hôtes, le MDP peut être le moyen de diffuser dans toute l'économie de ces pays un coût d'opportunité aux émissions de GES. Cela ferait entrer progressivement ces pays dans la logique internationale de la prévention du risque climatique, tout en assurant les transferts financiers requis pour ne pas pénaliser leurs efforts de développement économique (Godard et Henry, 1998).

\section{Obtenir que les règles de l'après 2012 soient fondées sur de nouvelles bases, moins arbitraires pour la France et susceptibles d'être attractives pour les pays en développement}

Il est important de préparer dès maintenant les conditions de l'après 2012. Au minimum, il faut éviter que la répartition des objectifs adoptée pour la période 2008-2012, peu satisfaisante sur le terrain de l'équité et désavantageuse pour la France, ne puisse faire précédent pour la suite. Le principal enjeu est de trouver des règles qui permettent aux pays en développement de rejoindre le groupe des pays de l'Annexe 1 ayant souscrit des engagements chiffrés contraignants. Ces règles devraient être suffisamment attractives pour les nouveaux entrants sans être pénalisantes pour les pays qui ont déjà souscrit à de tels objectifs.

Avant de faire corps avec les propositions de l'Union européenne, la France avait proposé un schéma de répartition des objectifs chiffrés de réduction des émissions qui prenait en compte les émissions par habitant et s'organisait autour d'un concept de convergence à très long terme des quotas d'émission par habitant de chaque pays. Ce critère peut être défendu sur le terrain de l'équité, bien qu'il ne soit pas le seul à être légitime sur ce terrain, et il est $a$ priori attractif pour les pays en développement les plus peuplés. Il peut cependant être jugé trop unilatéral. La France pourrait opportunément rappeler que dans l'annexe du traité de Maastricht relatif à la Banque centrale européenne, il a été jugé équitable par tous les pays de l'Union que chaque pays puisse souscrire au capital de cette banque au prorata de deux critères, comptant chacun pour moitié : le PIB et la population. Ce précédent pourrait avantageusement inspirer la formulation des règles de répartition des efforts après 2012.

\section{Références bibliographiques}

Azar, C. (1998), «Are Optimal $\mathrm{CO}_{2}$ Emissions Really Optimal ? Four Critical Issues for Economists in the Greenhouse », Environmental and Resource Economics, 11 (3-4), pp. 301-315.

Bruce, J.P., Lee, H. et Haites, E.F. (dir.) (1997), Le changement climatique. Dimensions économiques et sociales. Contribution du groupe de travail III au Deuxième rapport d'évaluation du Groupes d'experts intergouvernemental sur l'évolution du climat. Paris, Ed. 4D, diffusion La documentation Française, $546 \mathrm{p}$.

Bureau, D. et Hourcade, J.-C. (1998), «Les dividendes économiques d'une réforme fiscale écologique », in Conseil d'analyse économique.- Fiscalité de l'environnement. Paris, la Documentation française, Collection des Rapports du CAE auprès du Premier Ministre, juillet, 41-81. 
Cline, W. (1992), The Economics of Global Warming. Washinton DC, Institute for International Economics.

Cohen de Lara, M. et Dron, D. (1998), Évaluation économique et environnement dans les décisions publiques Rapport au ministre de l'Environnement. Paris, la Documentation française, Collection des rapports officiels, $416 \mathrm{p}$.

Commissariat Général du Plan (1993), L'économie face à l'écologie. Paris, Ed. La Découverte et La documentation Française.

Commissariat général du plan (1998).- Énergie 2010-2020 - Rapport final de l'Atelier 'Trois scénarios énergétiques pour la France'. Paris, Cgp, septembre, 310 p.

Fankhauser, S. (1995), Valuing Climate Change, London, Earthscan.

Godard, O. (1993), «Stratégies industrielles et conventions d'environnement : de l'univers stabilisé aux univers controversés », in INSEE.- Environnement et économie, INSEE-Méthodes (39-40), pp. 145-174.

Godard, O. (dir.) (1997a), Le principe de précaution dans la conduite des affaires humaines. Paris, Ed. de la Maison des sciences de l'Homme et INRA-Editions.

Godard, O. (1997b), «Les permis négociables et la Convention sur le climat: de l'expérience américaine aux enjeux de l'harmonisation », Revue de l'Énergie, (491), octobre, pp. 606-622.

Godard, O. et Henry, C. (1998), «Les instruments des politiques internationales de l'environnement: la prévention du risque climatique et les mécanismes de permis négociables ».- In Conseil d'analyse économique.- Fiscalité de l'environnement. Paris, la Documentation française, Collection des Rapports du CAE auprès du Premier Ministre, juillet, pp. 83-174.

Gollier, C. (1998), «Actualisation du long terme », Revue de l'énergie, (496), mars-avril.

Ha-Duong, M., Grubb, M.J., and Hourcade, J.-C. (1997), « Influence of socioeconomic inertia and uncertainty on optimal CO2-emission abatement », Nature, 390, pp. 270-273.

Hope, C. and Maul, P. (1996), «Valuing the Impact of CO2 Emissions », Energy Policy, 24, (3), pp. 211-219.

Hourcade, J.-C. (1997), «Précaution et approche séquentielle de la décision face aux risques climatiques de l'effet de serre » in Godard, O. (dir.) (1997a), op.cit., pp. 259-294.

Manne, A. and Richels, R. (1995), «The Greenhouse Debate: Economic Efficiency, Burden Sharing and Hedging Strategies », The Energy Journal, 16, (4), pp. 1-37.

Nordhaus, W. (1991), «To Slow or Not to Slow : the Economics of the Greenhouse Effect», The Economic Journal, 101, July, pp. 920-937.

Parry, I., Williams III, R.C., Goulder, L.H. (1996), When Can Carbon Abatement Policies Increase Welfare? The Fundamental Role of Distorted Factor Markets. Washington D.C., Resources for the Future, December.

Roqueplo, P. (1993), Climats sous surveillance. Limites et conditions de l'expertise scientifique. Paris, Economica.

Tol, R.S.J. (1995), «The Damage Costs of Climate Change. Towards More Comprehensive Calculations », Environmental and Resource Economics, 5, (4), June, pp. 353-374.

Wigley, T.M. (1997), «Implications of Recent CO2 Emission-limitation Proposals for Stabilization of Atmospheric Concentrations », Nature, 390, 267-270.

Wigley, T.M., Richels, R. and Edmonds, J.A. (1996), «Economic and Environmental Choices in the Stabilisation of Atmospheric CO2 Concentrations », Nature, 379, 240-243. 